\section{CONTRIBUTION OF INTERVENTIONAL RADIOLOGY IN PEDIATRICS}

E. Botsa ${ }^{1}$, I. Koutsogiannis ${ }^{2}$, E. Sotiropoulou ${ }^{3}$, K. Stathopoulos ${ }^{3}$, L. Thanos ${ }^{3}$

${ }^{1}$ Agia Sofia Children's Hospital, ${ }^{2}$ Aglaia Kyriakou Children's Hospital, ${ }^{3}$ Sotiria General Hospital, Athens, Greece

Background and aims: Interventional radiology is established as a diagnostic and therapeutic method in adults. Since lately it's use in children was limited due to lack of clinical experience. The aim is to detect the effectiveness and safety of invasive radiology as a method of diagnosis and treatment in pediatric patients.

Methods: A total of 138 children underwent a CT guided invasive procedure during the last 3 years. Their age ranged from 3-14 years with a mean age of 8 years. Most of our diagnostic cases referred to liver, kidney, lung, mediastinum and bone percutaneous biopsies (82 patients) and diagnostic drainages. Therapeutic approaches referred to drainage of abscesses or fluid collections (32 patients), radiofrequency ablation of osteoid osteomas (17 patients), radiofrequency ablation of liver (4 patients) and lung (3 patients) malignancies.

All procedures were performed under sedation. Depending on the kind of interventional procedure, the age and the figure of each patient different material was used. Biopsies were performed with needles $18 \mathrm{G}$ and cutting trocar system $14 \mathrm{G}$ with a needle of $18 \mathrm{G}$, drainages with drainage catheters trocar, pigtail 7 or $8 \mathrm{~F}$, and RFAs with spiral electrodes.

Results: Adequate diagnostic cytologic material was obtained in $90.2 \%$ of the diagnostic procedures. Therapeutic procedures were successful in $96 \%$ of our patients. None major complication was obtained in our patients.

Conclusions: Interventional radiology is a minimal invasive, simple, effective and safe method of diagnosis and treatment for children with no major complications. It results in low morbidity and short term hospitalization of pediatric patients.

\section{NBN GENE MUTATION I171V AS A POTENTIAL RISK FACTOR FOR CHILDREN MALIGNANCIES}

J. Nowak ${ }^{1}$, M. Mosor ${ }^{1}$, K. Nowicka ${ }^{1}$,

J. Rembowska ${ }^{1}$, D. Januszkiewicz ${ }^{1,2}$

${ }^{1}$ Institute of Human Genetics, Polish Academy of Sciences, ${ }^{2}$ Department of Paediatric Oncology, Haematology and Transplantology, University of Medical Sciences, Poznan, Poland

NBN (former NBS1) gene is considered as one of the low to moderate cancer susceptibility gene. At least four germinal NBN mutations have been found in several malignancies in adults. In our studies we observed the high incidence of germinal mutation I171V of NBN gene in breast, colorectal, larynx cancer and in multiple primary tumors. In present study we would like to answer the question whether I171V germinal mutation of NBN gene may constitue risk factor for solid tumors and acute leukemias in children. The frequency of this mutation has been analysed in patients with neuroblastoma $(n=66)$, Wilms' tumor $(n=54)$, medulloblastoma $(n=57)$, rhabdomyosarcoma $(n=82)$ and acute lymphoblastic leukemia $(n=173)$ hospitalized in Pediatric Oncology, Hematology and Bone Marrow Transplantation Department Poznań University of Medical Sciences in the years 1987 and 2010. 2947 anonymous blood samples collected on Guthrie cards drawn from the newborn screening program of the Wielkopolska voivodship have been used as controls. The study was approved by the Ethic Committee of the University of Medical Sciences in Poznań. All the patients and population controls came from the same geographical region. I171V mutation of the NBN gene has been observed in 7 children with acute lymphoblastic leukemia and in 5 control cases. Among children with solid tumors only in one child with medulloblastoma I171V variant has been found. In conclusion I171V germinal mutation in contrary to adults can't be considered as a risk factor for children malignancies except acute lymphoblastic leukemia.

Supported in part by Grant NN 407016235. 